\title{
Prenatal and postnatal development of laterally connected orientation maps
}

\author{
James A. Bednar and Risto Miikkulainen \\ Department of Computer Sciences, The University of Texas at Austin, Austin, TX 78712 \\ \{jbednar, risto\} ecs. utexas.edu
}

\begin{abstract}
Both environmental and genetic factors interact to produce the orientation maps found in the primary visual cortex of adult mammals. However, it is not clear how this interaction occurs during development, or whether both factors are crucial. Previous computational models have focused on either environmentally driven or genetically driven development alone. In contrast, we show that a two-stage model of development can account for a wider range of experimental data. The model explains how environmental and genetic information can be incorporated into the same neural hardware, using a common set of learning mechanisms. Our results suggest that while either environmental or genetically driven development is sufficient for maps and selectivity to form, prenatal activity speeds up early development and makes it more robust against environmental variation.
\end{abstract}

Key words: Orientation maps, Spontaneous activity, Cortical modeling, Nature-nurture

\section{Introduction}

Experiments in mammals such as cats, ferrets, and monkeys have shown that the orientation map patterns and receptive fields in primary visual cortex (V1) are produced by an interaction between environmental and genetic factors. Genetic influences are clear when measuring neural responses in newborns, before any visual experience. Even at or before natural eye opening, orientation-selective cells and orientation maps can be detected in newborn kittens and ferrets $[5,8,9,11,12]$. The overall shape of the orientation map changes very little during subsequent normal visual experience [9, 11]. Based on this evidence, one might conclude that orientation maps are largely genetically specified.

At the same time, it is clear that altering the visual environment can have dramatic effects on how orientation-selective neurons and maps develop. For instance, if kittens are raised in environments consisting of only one orientation of contour (e.g. vertical lines) during a critical period, an abnormally large number of their V1 neurons become responsive to vertical orientations [4]. Kittens raised in this way develop orientation maps that with larger area devoted to the overrepresented orientation [14]. Cats whose visual environment was even more abnormal, e.g. with eyelids sutured shut during development, have few orientation-selective neurons at all in V1 [5, 11]. Even in normal adult animals, the distribution of orientation preferences is slightly biased towards horizontal and vertical [7, 10], mirroring the distribution of orientations in normal visual environments [17]. Such a bias is consistent with neurons learning orientation selectivity from the environment. Orientation selectivity also improves greatly after birth, and 
Fig. 1. HLISSOM model of orientation map development. The model is a hierarchy of sheets of neural units, modeling the early visual pathway. There are two sheets of LGN units. Each unit receives input from its circular receptive fields (RF) on the photoreceptors. Connections to a sample photoreceptor RF are plotted for two units. Similarly, neurons in V1 have circular RFs on both LGN sheets; connections to two of the RFs are shown. V1 neurons also have lateral excitatory (small dotted circle) and lateral inhibitory (large dashed circle) connections to their neighbors. Input patterns are drawn in a grayscale bitmap on the photoreceptor sheet, and the ON and OFF LGN units compute their activity. Once all LGN units have been activated, initial V1 activity is computed from the LGN responses, and the activity then spreads laterally within V1. Both afferent and lateral V1 weights are adapted when the activity settles.

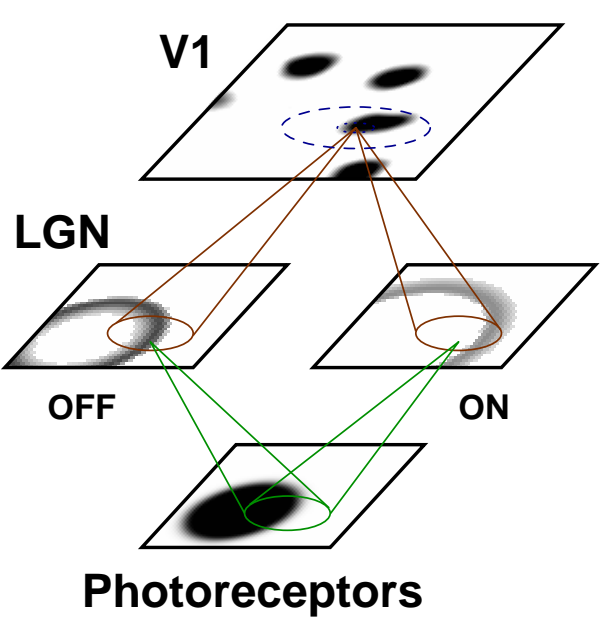

the maps become smoother and more well organized [9, 11]. Based on the postnatal experiments, one might conclude that orientation maps develop through visual experience alone.

Taken together, the evidence indicates that both genetic and environmental influences interact to produce the adult orientation map. However, important questions remain. How does this interaction actually occur? Could adult-like maps develop from environmental or genetic cues alone, or are both necessary? These questions are difficult to answer through biological experiments. Computational modeling, however, can lead to valuable insights, because it is easy to separate environmental and genetic influences in computational experiments. Existing models have been used to simulate how orientation maps can develop from visual input alone (e.g. natural images; [6]) or genetic factors alone, such as spontaneous neural activity (e.g. noise [13]; for review of existing models of each type, see [16].) However, models have not yet shown how V1 can have an initial map at birth that becomes smoother and more selective due to postnatal visual experience, while retaining the original map shape. The visually driven and internally driven models also differ in many ways besides the source of activity, and thus it has been difficult to determine whether the activity patterns alone account for any differences between the results.

In this paper we construct a single model to show how an initial map can develop from spontaneous neural activity, then be refined through visual experience to reflect the environment. The model develops orientation maps and selective neurons for a very wide range of training patterns. This result suggests that the processing implemented in orientation maps is very general, and does not need to be encoded specifically in the genome. The role of spontaneous activity may primarily be to speed up development so that even newborns have functional neural processing. This ability may make development more predictable in unusual environments, and allow higher levels to organize sooner.

\section{HLISSOM model}

The simulations are based on the HLISSOM model [1], a version of the LISSOM model [15] extended to support natural images by modeling the LGN. The architecture is shown in figure 1, and will be briefly reviewed below. (For more details, see [3].) The model consists of a hierarchy of two-dimensional sheets of neural units modeling different areas of the visual system: a sheet of retinal photoreceptors, a pair of sheets of LGN units (ON-center and OFF-center), and a sheet 
of cortical units ("neurons") representing V1. Because the focus is on the two-dimensional organization of V1, each neuron corresponds to a vertical column of cells through the six anatomical layers of the cortex.

The input to the model consists of a series of activity patterns on the sheet of photoreceptors, such as grayscale photographic images. Each LGN unit $(i, j)$ computes its response $\eta_{i j}$ as a scalar product of a fixed weight vector and its receptive field (RF) on the photoreceptor sheet:

$$
\eta_{i j}=\sigma\left(\sum_{\rho a b} \gamma_{\rho} X_{\rho a b} w_{i j, \rho a b}\right),
$$

where $\sigma$ is a piecewise linear sigmoid activation function, $X_{\rho a b}$ is the activation of input unit $(a, b)$ in $\mathrm{RF} \rho$ at timestep $t, w_{i j, \rho a b}$ is the corresponding weight value, and $\gamma_{\rho}$ is a constant scaling factor. Each V1 neuron computes its initial response like that of an LGN unit, except that $\rho$ takes two values, specifying the RFs on the ON and OFF LGN sheets. After the initial response, the V1 activity settles through short-range excitatory and long-range inhibitory lateral interaction:

$$
\eta_{i j}(s)=\sigma\left(\sum_{\rho a b} \gamma_{\rho} X_{\rho a b}(s-1) w_{i j, \rho a b}\right),
$$

where $\rho$ specifies one of the afferent RFs on the LGN sheets or the lateral excitatory or inhibitory weights to $\mathrm{V} 1, \gamma_{\rho}$ is a constant scaling factor for each $\rho$ (negative for inhibitory lateral weights), and $X_{\rho a b}(s-1)$ is the activation of input unit $(a, b)$ during the previous settling step. The V1 activity pattern starts out diffuse, but within a few iterations of equation 2 , converges into a small number of stable focused patches of activity, or activity bubbles. After the activity has settled, the connection weights of each V1 neuron are modified. All V1 weights adapt according to the Hebb rule, normalized so that the sum of the weights from each type of RF $\rho$ is constant for each neuron $(i, j)$ :

$$
w_{i j, \rho a b}(f+1)=\frac{w_{i j, \rho a b}(f)+\alpha_{\rho} \eta_{i j} X_{\rho a b}}{\sum_{a b}\left[w_{i j, \rho a b}(f)+\alpha_{\rho} \eta_{i j} X_{\rho a b}\right]},
$$

where $\eta_{i j}$ stands for the activity of neuron $(i, j)$ in the final activity bubble, $w_{i j, \rho a b}(f)$ is the connection weight from the previous fixation, $\alpha$ is the learning rate for each type of connection, and $X_{\rho a b}$ is the presynaptic activity. The larger the product of the pre- and post-synaptic activity $\eta_{i j} X_{\rho a b}$, the larger the weight change. At long distances, few neurons have correlated activity, so most long-range connections eventually become weak. The weakest connections are eliminated periodically, resulting in patchy lateral connectivity similar to that observed in V1.

For the experiments reported in this paper, the $36 \times 36$ ON-center and the $36 \times 36$ OFF-center LGN sheets received input from a $54 \times 54$ photoreceptor sheet. Each ON/OFF unit had a fixed difference-of-Gaussians RF within the photoreceptor array. Initially, the afferent weights of the $96 \times 96$ V1 neurons were random, and the lateral weights had a smooth circular Gaussian profile. The learning parameters were the same as in our earlier V1 orientation model [2], scaled for this cortex size and input patterns (see [1] for more details).

\section{Experiments}

To study how prenatal and postnatal learning together produce the adult organization, we simulated a two-stage process of development. In the prenatal phase of 1000 input presentations, 


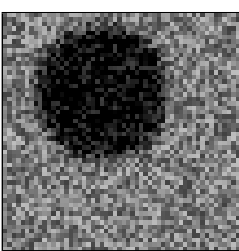

(a) Noisy Disks

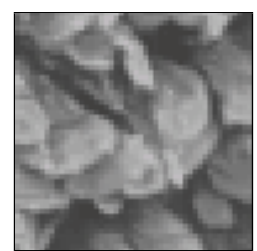

(b) Nature

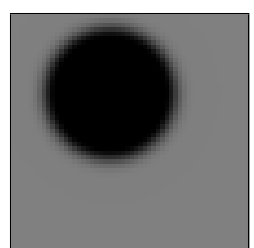

(c) Disks

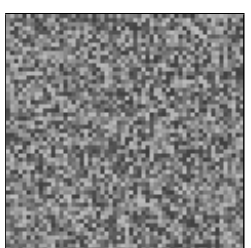

(d) Noise

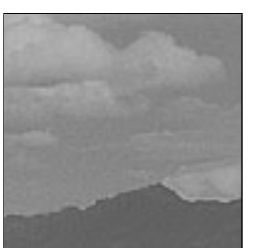

(e) Landscapes

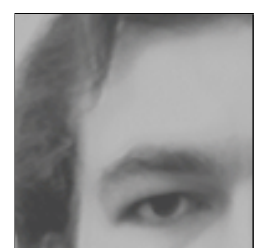

(f) Faces

Fig. 2. Training patterns. The network was trained prenatally on 1000 noisy, disklike activity patterns, as in $(a)$, followed by 9000 images of natural objects and natural scenes, as in $(b)$. The prenatal training is intended to model retinal waves, which have spatially coherent patches. We also tested a series of control patterns, including the disks alone $(c)$, random noise alone $(d)$, horizontally biased images $(e)$, and vertically biased images $(f)$. Results with these other patterns show what features of the prenatal and postnatal activity are most important.

input patterns consisted of noisy patterns of neural activity (figure $2 a$ ). These patterns were chosen to match retinal waves, which are the best-characterized source of spontaneous activity in early development. However, they can also represent any other spontaneous activity that includes large patches that are spatially coherent. The patterns consisted of uniform random noise added to a large "disk" of activity representing a local patch of highly responding neurons (a retinal wave). Figure $2 a$ shows an example. For the 9000 iterations in the postnatal phase, we used images of natural objects and scenes, modeling postnatal visual experience (see example image in figure $2 b$ ).

Figure 3 shows that this network first develops an orientation map through spontaneous activity, then gradually refines it based on experience with natural images, without changing the overall shape of the map. The orientation preference distribution is also biased towards horizontal and vertical, as seen in ferret visual cortex [10]. These results are the main novel component of this work. To our knowledge, only one other computational model has been tested in this way; the postnatal training was found to entirely rearrange the map in that model [6], unlike in animals.

In further experiments, we explored how different pattern types can change the map organization. These three experiments act as controls for the main results, helping to determine what features of the model are crucial. Details are presented in [1], but the results are summarized here. First, we tested different postnatal training conditions, to see how different environments can change the outcome. Skewed data sets were indeed reflected in the maps, as found for ferrets [14]. For instance, for the Landscapes dataset (figure $2 e$ ), containing many horizontal contours, the orientation histogram became biased towards horizontal. For the Faces dataset (figure $2 f$ ), more neurons became tuned to vertical orientations. These findings replicate the experimental results from animals raised in biased environments, and suggest that the postnatal component of training is important because it allows the map to adapt to the actual environment of the animal.

Second, we tested different patterns of prenatal training activity, to determine what features of the noisy disks were crucial. Both the disks alone (figure $2 c$ ) and random noise alone $(2 d)$ resulted in orientation maps with locally coherent patches of orientation-selective neurons. However, the receptive fields in the Disks case were much more selective than in typical newborn neurons. Thus including noise was crucial for obtaining realistic RFs. Yet for uniform random noise alone, the RFs were poorly selective, and primarily included RF types rarely reported in 


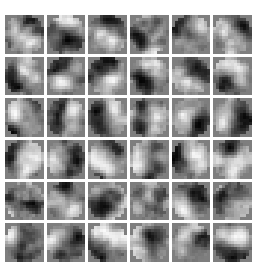

(a) Newborn RFs

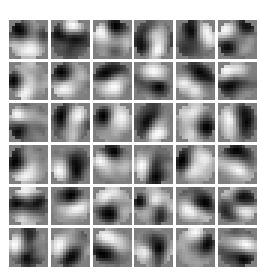

(b) Adult RFs
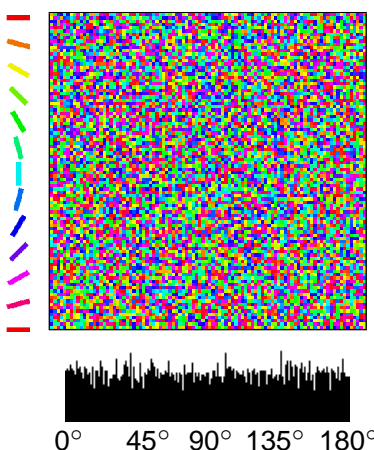

(c) Initial map
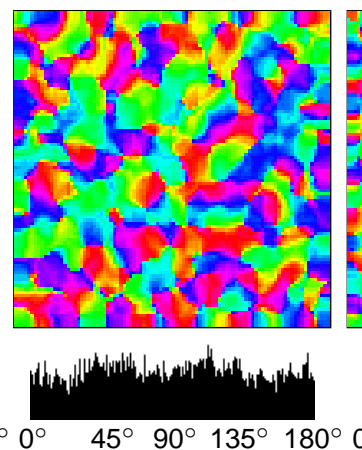

(d) Newborn map

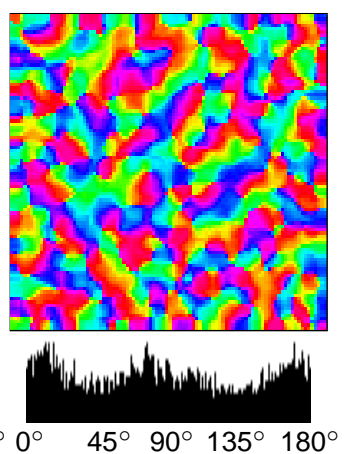

(e) Adult map

Fig. 3. Two-stage development of the orientation map. These plots show receptive fields (RFs) and orientation maps at the different developmental stages in the model. The RFs of each neuron were initially random, but by the end of prenatal training, neurons have multi-lobed receptive fields, as found in animals. Plot $(a)$ shows RFs for every sixteenth neuron in the newborn map (a $6 \times 6$ array), with black areas indicating net OFF-cell preference, white areas indicating net ON preference, and medium gray indicating no preference. The RFs become smoother and more selective with visual experience, but many of them keep the same overall shape $(b)$. The remaining plots summarize these RFs for the entire network as orientation maps, at the start of the simulation, $(c)$, after prenatal training $(d)$, and after postnatal training $(e)$. In each orientation map, each neuron is colored using grayscale coding of its preferred orientation, according to the key between $(a)$ and $(c)$. (Color versions of these figures are available at http://nn.cs. utexas.edu.) At first, the orientation preferences are random and very weak, due to the random RFs. After prenatal training, an orientation map has emerged, consisting of patches of neurons that prefer each orientation $(d)$. As subsequent training with natural images increases the number of selective neurons, the positions of many of the orientation-selective patches are preserved (e). The orientation histograms below each map show that the initial and newborn maps have approximately flat distributions, but that the adult map has significantly more units that prefer horizontal $\left(0^{\circ}\right.$ and $180^{\circ}$ ) and vertical $\left(90^{\circ}\right)$, as in animals and in the visual environment. These results show how genetic and environmental factors could interact to produce adult orientation maps and orientation-selective neurons.

experimental studies of V1 (e.g. crosses). The orientation map was also less well organized than typical newborn maps. These results suggest that both spatially uncorrelated noise and spatially correlated activity determine how the prenatal map develops.

Third, we tested whether the prenatal training was crucial for developing orientation maps and selectivity, by training an initially random map only on natural images. Although the details of the final map organization differed from the normal case, the overall properties were similar. Thus prenatal training is not required for a functional map to develop from natural image input.

\section{Discussion and future work}

The results presented in this paper show how environmental and genetic factors can interact to produce adult-like orientation maps. Both factors are crucial for explaining the experimental data, but the development of orientation maps and selectivity is very robust, and maps can develop from a wide range of possible input patterns. The specific distribution of orientation preferences in the adult map reflects the distribution of orientations in the activity patterns, allowing the map to adapt to the environment.

Interestingly, prenatal training does not appear to be crucial for obtaining a well-organized final 
map, at least in normal environments. Thus it is not yet clear what roles the spontaneous activity and the maps found in newborns perform. They may help ensure, however, that maps develop normally in a wider range of possible environments. Prenatal training may also allow V1 to mature more quickly, which could be important for organizing higher levels of the visual system. Future studies will focus on how to distinguish between different types of internally generated activity from the resulting maps, in hopes of narrowing down the specific patterns responsible for the initial orientation maps in newborns.

\section{Conclusion}

The HLISSOM model shows how environmental and genetic factors can interact to produce adult orientation maps. Either factor alone is sufficient for developing the maps, but both are necessary to explain how newborns can have maps initially yet adapt to the environment. Orientation maps and selectivity appear to be very robust outcomes of developmental processes, and prenatal activity may be important primarily as an optimization for development speed and predictability.

\section{Acknowledgements}

Supported in part by the National Institutes of Mental Health under Human Brain Project grant 1R01-MH66991, and by the National Science Foundation under grant IIS-9811478.

\section{References}

[1] Bednar, J. A. (2002). Learning to See: Genetic and Environmental Influences on Visual Development PhD thesis, Department of Computer Sciences, The University of Texas at Austin. Tech. Report AI-TR-02-294.

[2] Bednar, J. A., and Miikkulainen, R. (2000). Tilt aftereffects in a self-organizing model of the primary visual cortex. Neural Computation, 12(7):1721-1740.

[3] Bednar, J. A., and Miikkulainen, R. (2003). Learning innate face preferences. Neural Computation, 15(7):1525-1557.

[4] Blakemore, C., and Cooper, G. F. (1970). Development of the brain depends on the visual environment. Nature, 228:477-478.

[5] Blakemore, C., and van Sluyters, R. C. (1975). Innate and environmental factors in the development of the kitten's visual cortex. Journal of Physiology(London), 248:663-716.

[6] Burger, T., and Lang, E. W. (1999). An incremental Hebbian learning model of the primary visual cortex with lateral plasticity and real input patterns. Zeitschrift für Naturforschung C, 54:128-140.

[7] Chapman, B., and Bonhoeffer, T. (1998). Overrepresentation of horizontal and vertical orientation preferences in developing ferret area 17. Proc. of the National Academy of Sciences, USA, 95:2609-2614.

[8] Chapman, B., and Stryker, M. P. (1993). Development of orientation selectivity in ferret primary visual cortex and effects of deprivation. Journal of Neuroscience, 13(12):5251-5262.

[9] Chapman, B., Stryker, M. P., and Bonhoeffer, T. (1996). Development of orientation preference maps in ferret primary visual cortex. Journal of Neuroscience, 16(20):6443-6453.

[10] Coppola, D. M., White, L. E., Fitzpatrick, D., and Purves, D. (1998). Unequal representation of cardinal and oblique contours in ferret visual cortex. Proc. of the National Academy of Sciences, USA, 95(5):2621-2623.

[11] Crair, M. C., Gillespie, D. C., and Stryker, M. P. (1998). The role of visual experience in the development of columns in cat visual cortex Science, 279:566-570.

[12] Gödecke, I., Kim, D. S., Bonhoeffer, T., and Singer, W. (1997). Development of orientation preference maps in area 18 of kitten visual cortex. European Journal of Neuroscience, 9(8):1754-1762.

[13] Linsker, R. (1986). From basic network principles to neural architecture: Emergence of orientation columns. Proc. of the National Academy of Sciences, USA, 83:8779-8783.

[14] Sengpiel, F., Stawinski, P., and Bonhoeffer, T. (1999). Influence of experience on orientation maps in cat visual cortex Nature Neuroscience, 2(8):727-732.

[15] Sirosh, J., and Miikkulainen, R. (1994). Cooperative self-organization of afferent and lateral connections in cortical maps Biological Cybernetics, 71:66-78.

[16] Swindale, N. V. (1996). The development of topography in the visual cortex: A review of models Network - Computation in Neural Systems, 7:161-247.

[17] Switkes, E., Mayer, M. J., and Sloan, J. A. (1978). Spatial frequency analysis of the visual environment: Anisotropy and the carpentered environment hypothesis. Vision Research, 18(10):1393-1399. 